\title{
Adaptation in motion perception: Alteration of induced motion
}

\author{
HANS WALLACH \\ Swarthmore College, Swarthmore, Pennsylvania 19081 \\ JOSHUA BACON \\ Tufts University, Medford, Massachusetts 02155
}

and

\author{
PAUL SCHULMAN \\ State University of New York, College of Technology at Utica/Rome, Utica, New York 13502
}

\begin{abstract}
Prolonged exposure to a condition that causes induced motion was found to diminish this effect. The extent of a horizontal induced motion was measured by obtaining estimates of the direction of the apparent oblique path that resulted when a spot was visible on a horizontally moving pattern and was therefore in horizontal induced motion and, at the same time, moved vertically. Because the horizontal component of the perceived motion path represented the induced motion, the slope of the path measured the extent of the induced motion. After a 10-min exposure to induced motion, the apparent motion path was steeper; the mean change corresponded to a $15 \%$ smaller extent of the induced motion. Results were obtained that argue that this effect is not due to a diminished horizontal motion of the pattern but amounts to a smaller motion-inducing effect. The experiments were meant to support the view that the perceptual process that underlies induced motion is learned.
\end{abstract}

Duncker (1929) made it clear that visual motion perception can be based on two radically different conditions. In object-relative displacement, the motion of an object is given through its displacement relative to its visual environment, and in subjectrelative displacement, the motion is given as a gradual change of the moving object's visual direction, its angular displacement. Two conditions of stimulation mediate angular displacement: pursuit movement which occurs when one follows the moving object with ones eyes and displacement of the image of the moving object across the retina which occurs when one looks at a stationary point in the visual field. Usually, object-relative and subject-relative displacements are present simultaneously. When the moving object is given in a homogeneous field, however, the motion is given only subject-relatively as an angular displacement. The stimulus condition by which object-relative displacement 'makes itself felt is the displacement of the retinal image of the moving object relative to the image pattern of its surround, a changing configuration of the pattern on the retina. Which one of the two partners of the relative displacement, the moving object or the

This work was supported by Grant 11089 from the National Institute of Mental Health to Swarthmore College, Hans Wallach, principal investigator. surround, is represented by a stationary retinal image appears to be irrelevant. Neither is it necessary that the subject-relative displacement of the moving object causes it to be perceived as moving. The motion of the object may be too slow to be perceived on the basis of its angular displacement, while it is perceived on the basis of configural change.

Configurational change represents only a relative displacement between an object and its surround. Why, then, is the object perceived as moving and the background as stationary when angular displacement is not a factor? In motion perception based on configurational change only, motion and immobility are assigned according to a rule formulated by Duncker: The surrounded object will be perceived to move and the surround will tend to be perceived as stationary. In the absence of such a spatial relationship, for instance, when the relative displacement is between one moving and a single stationary object only, the assignment of perceived motion and immobility is unpredictable (Mack, Fisher, \& Fendrich, 1975). Most of the time, configurational change will lead to veridical motion perception. Occasionally, however, it will produce perceived motion of a stationary object, namely, when a stationary object is seen in a surround that undergoes a translatory displacement. In that case, the stationary object appears to move in the direction opposite to that of the displacement of the surround. Because it operates according to 
Duncker's rule, configurational change serves as a veridical cue only when the object whose image is displaced relative to the image pattern of its surround is actually moving. If the object is physically stationary and configurational change results from the actual motion of the surround, the stationary object may appear to move nevertheless. It is as if this apparent motion were induced. Hence the name for such an illusory perceived motion is "induced motion."

Induced motion is not always experienced when one views a stationary object in a moving surround. It is difficult to predict when induced motion will fail. It may fail because the other motion cues, the ones that mediate angular displacement, are also present. They represent the state of the stationary object correctly and a cue discrepancy results between the cues for angular displacement and configurational change. ${ }^{1}$ The result of a cue discrepancy in perceptual experience is always hard to predict (Wallach, 1968).

Whether or not the rate of motion of the surround is above the threshold for motion based on angular displacement cues seems to matter little. When the motion of the surround is also perceived, the sum of the two experienced motions, the induced motion of the surrounded object and the correctly assigned motion of the surround, may be larger than the relative displacement between the two would warrant; perceiving the motion of the surround does not necessarily diminish the induced motion. This observation led Duncker to formulate the notion of separation of systems: conditions that cause perceived motion of the surround do not necessarily interfere with the induced motion that is based on configurational change between that surround and the stationary object.

Induced motion is not the only fact that makes it necessary to assume that configurational change is an independent cue in motion perception. There are two further facts that demonstrate the effectiveness of configurational change. They are concerned with motion speed. An object moving with constant velocity in a nearly empty field will appear to speed up when it approaches a visual landmark (Johansson, 1950). As it does, the rate of configurational change increases. Secondly, Brown's transposition principle of speed perception (Brown, 1931) can be understood only if configurational change is the dominant factor; angular displacement would produce veridical speed perception (Wallach, 1976, p. 92).

One may ask why there are two conditions that cause motion perception, that is, configurational change in addition to angular displacement. Angular displacement would obviously suffice. Besides, it provides a more reliable condition of stimulation; above threshold, it always leads to veridical motion perception when the subject's own state as to move- ment or rest is adequately given. And what brings about Duncker's rule that transforms mere relative displacements into perceived motion and immobility? Wallach $(1976$, p. 98 ) has proposed that both result from the simplest kind of learning, the substitution of one stimulus condition for another. Such learning could take place under the most frequently occurring condition for motion perception, namely, when a moving object is seen surrounded by stationary objects. Its displacement, mediated by angular displacement, would cause it to be perceived as moving. At the same time, its retinal image would be displaced relative to the images of the stationary objects, and the pairing of this condition with perceived motion of the object may cause it to become a stimulus for perceiving motion. Moreover, the image of the object seen in motion is surrounded by the images of objects that are perceived as stationary: thus, assigning motion and immobility according to Duncker's rule could be simultaneously acquired.

The idea that configurational change is a learned cue for motion perception suggested that it might be possible to modify it by perceptual adaptation. Where several cues determine the same perceptual property, as is the case with depth, distance, and tilt, adaptation can often be achieved by exposing the subject for some time to a discrepancy between such paired cues. This is usually done by spectacles that alter the stimulation that provides one of the paired cues. Adaptation consists here in a changed cue evaluation that compensates partially for the altered cue. ${ }^{2}$ In the case of motion perception, exposure to a cue discrepancy is possible without an artificial alteration of one of the cues. The condition that may produce induced motion provides itself a cue discrepancy. As stated, a stationary object and a surround that undergoes translatory displacement provides a configurational change that is a stimulus condition for perceiving the object in motion, while the conditions mediating angular displacement represent the object as stationary. Prolonged exposure to this condition might, then, diminish the effectiveness of configurational change in producing motion perception.

We tested for a diminished effectiveness of configurational change by exposing the subject to an arrangement that yielded induced motion. Presenting configurational change in isolation is impossible, since angular displacement is always associated with it. ${ }^{3}$ This being the case, induced motion, where configurational change is in conflict with the cues for angular displacement, seems preferable to normal motion, where the two conditions of stimulation cooperate. One of us had previously found that induced motion is always perceived when the object that is seen in a moving surround is not stationary but moves at right angles with the direction of the 
induced motion. If, for instance, a spot that is seen against a horizontally moving background moves objectively in a vertical direction, it will always be perceived to move obliquely; oblique motion is here the resultant of the objective vertical motion and the induced horizontal motion. Oblique motion will be perceived even when the objective vertical motion is not given by configurational change. This can be achieved by eliminating all landmarks that a vertically moving spot would encounter, that is, by having the pattern of the horizontally moving background consist exclusively of vertical lines. In that case, the objective vertical motion is given by angular displacement only, namely, by displacement of the retinal image of the spot followed by ocular pursuit movements. Such an arrangement also has the advantage that the magnitude of induced motion is easily measured. The tilt of the apparent motion path represents the proportion of the effect of configurational change to that of angular displacement. The larger the angle between the apparent motion path and the vertical, the direction of the objective motion of the spot, the stronger the amount of induced motion for a given proportion of the horizontal motion of the background and of the vertical motion of the spot.

Thus, our attempt to diminish induced motion by prolonged exposure to induced motion took the following form. An estimate of the tilt of the apparent motion path was obtained that was produced by an objectively vertical motion of a spot visible against a background that moved horizontally at the same rate. Then the subject observed a stationary spot on a horizontally moving background. Following this adaptation period, which lasted $10 \mathrm{~min}$, another estimation test was obtained. To avoid producing, in our adaptation period, a quasisensory adaptation that would manifest itself, for instance, as a motion aftereffect all motions had to be reciprocating. This, in turn, meant that we could not use displacements of constant velocity for the motion of the spot and of the background, inasmuch as reversing the motion direction required acceleration and deceleration in each excursion. Mainly because it is easy to produce, we used simple harmonic motion. If the induced-motion condition is fully effective, reversing the two motions always simultaneously and making the extent of the two excursions equal should produce a straight motion path with a slope of $45^{\circ}$.

\section{EQUIPMENT}

The moving background (screen) consisted of a sheet of white translucent plastic, $80 \mathrm{~cm}$ high and $50 \mathrm{~cm}$ wide, in a sturdy aluminum frame. The pattern of vertical lines was provided by 20 evenly spaced copper wires, $.8 \mathrm{~mm}$ thick and $2.5 \mathrm{~cm}$ apart, which were stretched between the two horizontal bars of the frame so that they rested against the plastic sheet. Rollers were attached on both sides to the bottom bar of the screen; they ran in a retaining slot which was attached to a table. This arrangement allowed the screen to move horizontally. Through a horizontai bar attached to the frame in the rear, the frame was connected to the end of a scotch yoke, which caused the reciprocating motion of the screen. The pin that provided the circular motion that drove the yoke back and forth was excentrically attached to the vertical shaft of a variable-speed (shunt) motor.

The moving spot was a disk of light, $5 \mathrm{~mm}$ across, that was projected on the translucent screen from the rear, from a plastic tube, into which a small lens, a circular aperture and a small light bulb had been inserted. The tube was attached at right angles to a horizontal shaft that could be turned̆ by a lever fastened to the shaft perpendicularly to the tube. This lever was coupled to a second scotch yoke so that the yoke's excursion tilted the tube up and down and thereby caused the vertical excursion of the light spot. The shafts of the two yokes were connected by a gear train. A clutch in one of the shafts permitted adjustment between the phases of the yokes. The excursions of the screen and of the light spot could be varied by changing the distances between the yoke pins and the shafts about which they revolved. In addition, the excursion of the light spot could be adjusted by changing the effective length of the lever that tilted the plastic tube and made the light spot move up and down. The center of the path of the light spot coincided with the center of the screen when it was in its middle position. The subject's head was held in position by a chin- and headrest that was so adjusted that his eyes were at the level of this center and $40 \mathrm{~cm}$ from the screen. At this vantage point, the vertical extent of the screen subtended an angle of $90^{\circ}$. When the vertical excursion of the light spot was $15 \mathrm{~cm}$, its ends were separated from the upper and lower bars of the frame by visual angles of $34.4^{\circ}$.

The room was dark except for a shielded light that illuminated the translucent sheet at a moderate level from the rear. Cloth curtains were attached to the vertical edges of the screen and extended $30 \mathrm{~cm}$ to either side. On the subject's right and within easy reach was a white rod visible against a dark background that was attached perpendicularly to a horizontal shaft, so that the subject could set the rod to any desired tilt. Each time the subject was ready to make a setting, the experimenter lit a shielded $15-\mathrm{W}$ bulb that illuminated the rod.

\section{EXPERIMENT 1}

Our first experiment demonstrated an effect of prolonged exposure to induced movement. For $10 \mathrm{~min}$, the subject looked at the stationary light spot while the screen with the vertical line pattern moved from side to side at a rate of 20 excursions $/ \mathrm{min}$. The length of the excursions was $15 \mathrm{~cm}$. Before and after the exposure period, a tilt estimation test was administered. Here the light spot moved up and down in phase with the motion of the screen, which moved at the same rate as during the exposure period; the light spot also covered a distance of $15 \mathrm{~cm}$ at each excursion. As previously explained, the subject experienced the spot moving on a tilted path. He first observed the motion of the spot for four excursions and then set the white rod to a tilt equal to the tilt of the observed motion path. He gave four such tilt estimates, two where the rod was initially in horizontal position and two starting with 
Table 1

Mean Tilt Estimates (in Degrees) Before and After 10-Min Exposure to Induced Motion and After a 10-Min Dissipation Period; $\mathrm{N}=11$

\begin{tabular}{lccccc}
\hline & $\begin{array}{c}\text { Pre } \\
\text { Exposure } \\
\text { I }\end{array}$ & $\begin{array}{c}\text { Post } \\
\text { Exposure } \\
\text { II }\end{array}$ & $\begin{array}{l}\text { Diff } \\
\text { I-II }\end{array}$ & $\begin{array}{c}\text { Post } \\
\text { Dissipation } \\
\text { III }\end{array}$ & $\begin{array}{l}\text { Diff } \\
\text { I-III }\end{array}$ \\
\hline Mean & 42.6 & 38.8 & $3.8^{*}$ & 37.4 & $5.2^{* *}$ \\
S.D. & 9.34 & 8.10 & 3.6 & 10.57 & 5.08 \\
\hline
\end{tabular}

${ }^{*} t(10)=3.51 ; p<.01{ }^{*} t(10)=3.39 ; p<.01$

the rod vertical. The average of the four tilt estimates became the subject's preadaptation score. After the exposure period, the test was repeated but the subject gave only two estimates. As a check on the rate of dissipation of the adaptation effect, the subject walked outside for 10 min following the postexposure test and then gave two further tilt estimates. Eleven undergraduates served as subjects.

Table 1 lists the mean settings of the tilt angles, with the vertical direction equal to $0^{\circ}$. The mean tilt angle before adaptation was $42.6^{\circ}$, quite close to the value of $45^{\circ}$ that should have resulted had induced motion been just as effective in causing the horizontal motion component as angular displacement was in causing the vertical component. This result and the corresponding one reported below for Condition A in Experiment 2 show that induced motion is virtually complete under these conditions. In addition to the horizontal components of the apparent path of the spot, the motion of the screen was also clearly seen. Therefore, we have here an indisputable instance where, in induced motion, the sum of the two experienced motions is larger than the given relative displacement would justify, since the two experienced motions, the apparent motion of the surrounded object (the horizontal component of the spot's path) and the correctly assigned motion of the surround, that is, of the screen, are in opposite directions. As mentioned above, such instances make it clear that the two simultaneous motion processes, the motion of the surround and the motion of the surrounded object, are independent of each other.

After adaptation, the mean apparent tilt angle was more vertical by $3.8^{\circ}$, a change that implied a shortening of the horizontal motion component by $12.6 \%$. The adaptation effect did not decline during the dissipation period.

\section{EXPERIMENT 2}

Two interpretations can be given for the effect of the adaptation condition. The effectiveness of configurational change in causing induced motion may be diminished, and that is the result of prolonged exposure to induced motion we were looking for.
But is also possible that prolonged exposure to the reciprocating horizontal displacements of the screen had the effect that this condition of stimulation caused diminished horizontal motion perception, and this could have caused the decrease in the horizontal motion component that our tests measured. We also asked whether a diminished effectiveness of configurational change would be restricted to the horizontal displacements that had been involved in our adaptation exposure or whether it was general. An answer to this question could be obtained by introducing, in the test, landmarks for the vertical displacement of the light spot. This would add configurational change as a cue for the vertical displacement, which, in our normal test, is given only by angular change. If the diminished effectiveness of configurational change is general and applies to vertical displacement also, this should diminish the adaptation effect when it is measured with the tilt estimation method, provided that here, too, configurational change is the dominant cue. To provide the landmarks for vertical displacement, the vertical line pattern on the screen was changed into a square pattern by adding a grid of horizontal wires. Finally, we wanted to know whether the effect of our adaptation condition would make itself felt also when the subject did not fixate the moving light spot but followed, with his eyes, the horizontal motion of the screen.

\section{Procedure}

Our first experiment was repeated four times with four different test conditions (Figure 1). In Condition A, the tests were identical with those of Experiment 1, except that in the postadaptation

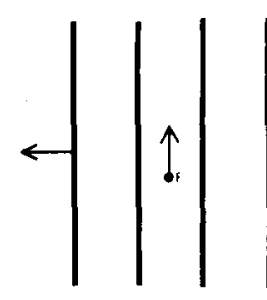

A

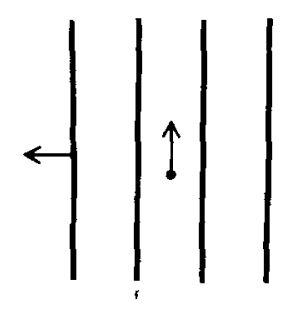

C

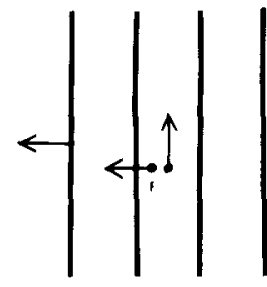

B

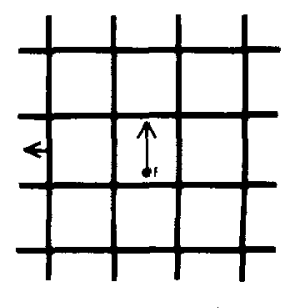

D
Figure 1. The four test conditions of Experiment 2. $F$ is the place of fixation. 
test the subject made four instead of two tilt estimates. This, we hoped, would diminish the variance of the postadaptation scores. This change was made possible by the finding in Experiment 1 that there was not rapid dissipation after the adaptation period. Four tilt estimates were made in all tests in Experiment 2. In test Condition B, a fixation mark was fastened to the moving screen and the subject was instructed to fixate it. In Condition $C$, the fixation mark was omitted but the subject was asked to keep his eyes on one of the vertical lines. In Condition D, as in Condition A, the subject was instructed to follow the moving light spot with his eyes, but the wire grid was added. Also, the horizontal excursion of the screen was shortened from 15 to $8.7 \mathrm{~cm}$ to make the tilt angle of the expected resultant $30^{\circ} .4$ This was done to avoid an apparent preadaptation tilt angle close to the diagonals in the square pattern. The subjects might have noticed that coincidence in the preadaptation trials and remembered it during the postadaptation test. Different subjects were used in the four test conditions; their numbers are listed in Table 2 .

\section{Results and Discussion}

The mean tilt settings that were obtained in the four test conditions, each followed by its standard deviation, are presented in Table 2, which also gives the mean pre- and postadaptation difference scores and their $t$ and $p$ values. Condition $A$ measured a highly significant adaptation effect that was in good agreement with the one obtained in Experiment 1. The change in the mean tilt estimate by $4.7^{\circ}$ means that the horizontal motion component was shortened by $15.2 \%$. The mean difference score of $4.7^{\circ}$ was also significantly different from the mean difference scores obtained in Conditions B $[\mathrm{t}(49)=3.06$, $\mathrm{p}<.01], \mathrm{C}[\mathrm{t}(35)=2.48, \mathrm{p}<.02]$, and $\mathrm{D}[\mathrm{t}(40)$ $=3.01, \mathrm{p}<.01]$. As in Experiment 1 , the mean tilt estimate before adaptation was close to the theoretical value of $45^{\circ}$, namely, $43.9^{\circ}$. This showed induced motion to be just as effective as real motion mediated by angular displacement.

The most important result was that of Condition D, where no adaptation effect was measured. It answers two of the questions we raised earlier. That no adaptation effect was here measured shows that the effect that was obtained in Condition A was not the result of a diminished perception of the horizontal motion of the screen. There is no reason why introducing horizontal lines into the test pattern should prevent a diminished horizontal motion perception from having its effect. This makes it very likely that the adaptation effect consists in a diminished effectiveness of the screen displacement to produce induced motion, in other words, that adaptation causes the configurational change to become less effective. The other question answered was whether this diminished effectiveress of configurational change is confined to horizontal displacements that the subject had adapted to or applies to all. The introduction of horizontal lines causes the vertical motion of the spot to be given as configurational change also, and the fact that no adaptation was measured shows that the diminished effectiveness of configurational change applies also to the objective vertical displacement of the spot. The adaptation diminishes the effects of the horizontal and of the vertical configurational change equally, and the tilt of the perceived motion path does not change.

It is interesting that introducing the horizontal lines in the test condition completely eliminated the effect of adaptation on the tilt of the perceived motion path, inasmuch as the vertical component of the spot's motion is given also as an angular displacement. If angular displacement had here been effective in mediating the vertical motion of the spot along with the configurational change provided by the presence of the horizontal lines, part of the effect measured in Condition A should have been obtained. The fact that it was not shows that configurational change is dominant not only when it is in conflict with angular displacement, as in the condition that yields induced motion, but also when the two conditions mediating motion perception operate in parallel.

Measuring virtually no adaptation effect with Condition B could have been due to the fixation mark serving as a landmark for the vertical motion of the spot, but this was not the case, as the negative result of Condition $C$ shows. In Condition $C$, the subject's eyes also followed the motion of the screen, but there was no fixation mark. ${ }^{5}$

The fact that the eyes tracked the screen rather than the spot that undergoes the induced motion must have been the reason why, in the Conditions $B$ and $C$, no adaptation was measured. In these two conditions, the spot is represented by a retinal image that is being displaced in two ways, horizontally, because the eyes turn to follow the motions of the screen, and vertically, due to the objective vertical motion of the spot. The two simultaneous displacements result in an oblique image path. One way to

Table 2

Mean Tilt Estimates (in Degrees) Before and After 10-Min Exposure to Induced Motion, with Their Standard Deviations and Mean Difference Scores

\begin{tabular}{|c|c|c|c|c|c|c|c|c|}
\hline \multirow[b]{2}{*}{$\begin{array}{l}\text { A } \\
\text { B } \\
\text { C } \\
\text { D }\end{array}$} & \multirow{2}{*}{$\begin{array}{l}\text { Test Conditions } \\
\text { eyes on spot, screen: lines } \\
\text { eyes follow screen, mark; screen: lines } \\
\text { eyes follow screen, no mark; screen: lines } \\
\text { eyes on spot; screen: squares }\end{array}$} & \multirow{2}{*}{$\begin{array}{l}\mathrm{N} \\
25 \\
26 \\
12\end{array}$} & \multicolumn{2}{|c|}{ Pre } & \multicolumn{2}{|c|}{ Post } & \multirow{2}{*}{$\begin{array}{l}\text { Diff } \\
4.7 \\
1.0\end{array}$} & 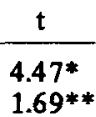 \\
\hline & & & $\begin{array}{l}43.9 \\
44.1 \\
38.3 \\
31.8\end{array}$ & $\begin{array}{l}8.5 \\
4.4 \\
4.5 \\
6.4\end{array}$ & $\begin{array}{l}39.2 \\
43.1 \\
37.8 \\
31.1\end{array}$ & $\begin{array}{l}9.3 \\
3.8 \\
5.2 \\
6.5\end{array}$ & & $\begin{array}{c}4.47^{*} \\
1.69^{* *} \\
.42 \\
.89\end{array}$ \\
\hline
\end{tabular}

${ }^{*} p<.001 \quad * * p>.05$ 
explain why Tests $B$ and $C$ did not measure the adaptation effect would be to assume that here the perception of the pathway tilt is based on the oblique retinal image path rather than on the combination of the perception of the vertical motion of the spot and of its horizontal induced motion. The oblique retinal image path would, of course, not be altered by the adaptation effect, and the tilt estimation test would, therefore, fail to measure adaptation.

This explanation is, however, based on a radical assumption; it implies that position constancy does not operate here. Position constancy is the compensation process that prevents image displacements that result from eye movements from causing perceived motion. Such compensations were first demonstrated by Mack (1970) for saccadic eye movements, and they have been investigated by Mack and Herman $(1973$, 1978) for pursuit eye movements. In Conditions B and C, however, the spot whose image is horizontally displaced by an eye movement also moves objectively, and this motion causes an additional vertical image displacement, with the two displacements resulting in the oblique image path. Compensation for the image displacement caused by eye movement would prevent the horizontal component of the image path from having an effect on the perceived motion path. Where saccadic eye movements are concerned, Mack (1970) showed that position constancy may correct for a component of an image path, but this has not yet been demonstrated for pursuit eye movements. It seems, nevertheless, likely that compensation for a displacement component occurs also when it is caused by a pursuit movement. In that case, the horizontal component of the spot's oblique image path would be rendered ineffective and the perceived motion of the spot would be vertical, except that now induced motion would take over and, just as in Condition $A$, produce the horizontal component of the perceived oblique motion path.

This interpretation would leave the failure of Test Conditions $B$ and $C$ to measure an adaptation effect yet to be explained. To account for that result, one might assume that the two induced motion processes, one that takes place when the surrounded object is fixated and the other when the eyes follow the moving surround, differ in nature, and that a diminished effectiveness of the first does not make itself felt when the test involves the second.
The other explanation of what goes on in Tests $B$ and $C$ is simpler. The sequence of compensation and induced motion is replaced by a single stimulus condition, the oblique path of the retinal image of the spot. More work is needed for deciding which of the two explanations is correct.

\section{REFERENCES}

Brown. J. F. The visual perception of velocity. Psychologische Forschung, 1931, 14, 199-232.

DunCKER, K. Über induzierte Bewegung. Psychologische Forschung, 1929, 12, 180-259.

Gogel, W. C., \& Kostow, M. The effect of perceived distance on induced movement. Perception \& Psychophysics, 1971, 10. 142-146.

Johansson, G. Configurations in the perception of velocity. Acta Psychologica, 1950, 7, 25-79.

MACK, A. An investigation of the relationship between eye and retinal image movement in the perception of movement. Perception \& Psychophysics, 1970, 8, 291-297.

MACK, A., Fisher, C. B., \& Fendrich, R. A re-examination of two-point induced movement. Perception \& Psychophysics, 1975. 17, 273-276.

Mack, A., \& Herman, E. Position constancy during pursuit eye movements: An investigation of the Filehne illusion. Quarterly Journal of Experimental Psychology, 1973, 25, 71-84.

Mack, A., \& Herman, E. The loss of position constancy during pursuit eye movements. Vision Research, 1978, 18, 55-62.

WALLACH, H. Informational discrepancy as a basis of perceptual adaptation. In S. J. Freedman (Ed.), The neuro-psychology of spatially oriented behavior. Homewood, Ill: Dorsey Press, 1968.

W AlLACH. H. On perception. New York, N.Y: Quadrangle/The New York Times Book Co., 1976.

\section{NOTES}

1. Subject-relative displacements, that is, angular change, can also have an effect on the magnitude of induced motion. This was demonstrated by Gogel and Koslow (1971).

2. For more discussion of adaptation based on cue discrepancy, see Wallach (1976), Chapter X.

3. Rendering angular displacement ineffective by employing velocities so slow that they are below the threshold for motion perception based on angular displacement seemed impractical.

4. In Experiment 1, the mean preadaptation tilt estimate had been close to $45^{\circ}$ when the excursions of the light spot and the screen were equal.

5. The absence of the fixation mark had an effect on the preadaptation tilt setting. The mean tilt estimates were here more vertical by $5.8^{\circ}$ than in Condition $B$, and this difference was highly significant $[\mathrm{t}(36)=3.75, \mathrm{p}<.001]$.

(Received for publication June 13, 1978; revision accepted September 22, 1978.) 